International Journal of Current Advanced Research

ISSN: O: 2319-6475, ISSN: P: 2319 - 6505, Impact Factor: SJIF: 5.995

Available Online at www.journalijcar.org

Volume 6; Issue 5; May 2017; Page No. 3993-3996

DOI: http://dx.doi.org/10.24327/ijcar.2017.3996.0414

\title{
EFFECT OF SLEEP POSITION ON DRY EYE DISORDER
}

\section{Zanak Sunil Dattatray ${ }^{1}$, Zanak Seema Dattatray ${ }^{2}$, Zanak Komal Dattatray ${ }^{3 .}$, Pranjali Pise $^{4}$ and Nikhil Pise ${ }^{5}$}

\author{
${ }^{1}$ Ophthalmology Department Government Medical College, Akola \\ ${ }^{2}$ Consultant Ophthalmologist. Zanak Netralay, Akola \\ 3,4,5Consultant Physician Zanak Netralay, Akola
}

\section{A R T I C L E I N F O}

\section{Article History:}

Received $24^{\text {th }}$ February, 2017

Received in revised form $15^{\text {th }}$ March, 2017

Accepted $14^{\text {th }}$ April, 2017

Published online $28^{\text {th }}$ May, 2017

\section{Key words:}

Multifactorial, cross-sectional, disease, staining, supine, corneal.

\begin{abstract}
A B S T R A C T
Purpose: Dry eye disease is a multifactorial disease with numerous well-documented risk factors. However, to date, sleep position has not been associated with this condition. After observing patients in our practice, we believe that the sleep position in some cases may significantly affect dry eye and meibomian gland dysfunction (MGD).
\end{abstract}

Methods: This is a single-centered, cross-sectional, noninterventional, institutional review board-approved, single-masked, nonrandomized study of 100 patients whose complaints were related to dry eye disease and a control group of 25 age-matched asymptomatic patients. Two questionnaires were used: one to analyze patients' sleep habits and the other to assess patients' Ocular Surface Disease Index. Dry eye severity was graded based on the MGD stage, fluorescein corneal staining and lissamine green staining, Schirmer 1 testing and clinical examination.

Results: A statistically significant difference was shown with back sleeping compared with left side sleeping using lissamine green staining (analysis of variance, $\mathrm{P}=0.005$ ). The Ocular Surface Disease Index score was also found to be elevated in patients who slept on their right or left side (36.4 and 34.1, respectively) as opposed to back sleepers (26.7) with $\mathrm{P}, 0.05$. There was no statistically significant correlation found between the sleep position and degree of MGD.

Conclusions: In addition to current treatment, patients who sleep on their side or face down might see a reduction in dry eye and MGD if they change their sleep pattern to the supine position.

Copyright $(2017$ Zanak Sunil Dattatray. This is an open access article distributed under the Creative Commons Attribution License, which permits unrestricted use, distribution, and reproduction in any medium, provided the original work is properly cited.

\section{INTRODUCTION}

\section{Study}

Meibomian gland dysfunction (MGD) is defined as chronic irregularity in the sebaceous glands of the eyelid characterized by duct obstruction and changes in gland secretion.1-3 There are several risk factors that have been known to exacerbate MGD. The International Workshop on Meibomian Gland Dysfunction categorized MGD risk factors into 3 broad areas: ophthalmic, systemic, and therapeutic.4 From these categories, some of the most common risk factors for MGD include chronic blepharitis (anterior and/or posterior), rosacea, allergic conjunctivitis, contact lens wear, Demodex folliculorum, high cholesterol levels, and aging.5,6 There are several environmental risk factors, such as geography, temperature, humidity, and certain visual tasks (such as computer use), that may impact MGD prevalence. 5 Yet, no

\section{*Corresponding author: Zanak Sunil Dattatray} Ophthalmology Department Government Medical College, Akola study has comprehensively looked into sleep position as a possible risk factor for dry eye and MGD. Several sleep studies have shown that side lying is the most common sleep position and the supine back) position is least common.6,7 In one study, $72 \%$ of 812 subjects in the study slept on the side, whereas only $11.2 \%$ slept in the supine position.6 Other studies 8 have shown similar findings. One study7 has even shown that elderly patients have a tendency to right-sided sleeping. Therefore, a minority of patients sleep on their back when compared with side or stomach sleeping positions.

Three studies have investigated the sleep position and dry eye disease (DED). One focused on nocturnal lagophthalmos in a high-airflow environment.9 This study was most relevant to the current study but lacked details about the degree of dry eye present. The authors evaluated 48 patients with nocturnal lagophthalmos in a high-airflow environment. Our study, in comparison, used more diagnostic parameters [Ocular Surface Disease Index (OSDI), lissamine staining, and Schirmer I testing] in an effort to better describe how the sleep position 
in the general population affects dry eye in general and MGD in particular. The second study focused on patients with sleep apnea, and the last study focused mainly on evaluating floppy eyelid syndrome (FES).10, 11 To our knowledge, those were the only studies that examined the sleep position and DED or MGD. Patients with keratoconus have a tendency to sleep with their face predominantly in the down position with pressure being placed on the face and eyelids.12 This longterm position for sleeping may predispose to FES and keratoconus. $12 \mathrm{We}$ believe that mechanical pressure placed by sleeping on one side or stomach may also increase MGD prevalence and ocular surface disease. We advise our patients to sleep in the supine position to avoid as little mechanical pressure to the eye and orbit as possible; this is in an effort to reduce the severity of their MGD and ocular surface disease, which should also provide a positive effect on their DED. 13,14 .

This study examined sleep patterns in patients with complaints of dry eye to see whether there exists any correlation between the sleep position and DED. Because of the multifactorial nature of DED and MGD, several factors in addition to the sleep position were evaluated and form the substance of this article.

\section{MATERIALS AND METHODS}

This is a single-centered, cross-sectional, noninterventional, institutional review board-approved, single-masked, nonrandomized study based out of the Ophthalmic Consultants of Government Medical College Akola and general practioner in Akola; Maharashtra; India.The study was conducted in accordance with the tenets of the Declaration of Helsinki. Institutional review board approval was obtained before commencing the study. A total of 125 patients volunteered to participate as study subjects and are the subject of this study. Patients were evaluated for symptomatic and clinical signs of DED and MGD by ophthalmologist using the diagnostic and staging criteria established by the American Academy of Ophthalmology.2 Consented patients underwent clinical evaluations including 2 questionnaires; OSDI and sleep survey,Snellen refraction, biomicroscopy (for lid, lash, and conjunctiva abnormalities), fluorescein corneal staining (FCS), Schirmer 1 testing, lissamine green staining (LGS), and intraocular pressure measurements. Meibomian glands were examined for expressibility and secretions, both in terms of quantity and consistency. If there was a diagnosis of MGD, it was staged 1 through 4 using the American Academy of Ophthalmology MGD diagnostic criteria.2

Evaluations were performed until 100 consecutive patients diagnosed with DED and MGD were recruited for the study. Inclusion criteria were a history of DED or OSDI scores of 25 or greater. Excluded were patients who did not have symptoms of dry eye, refused consent, and were currently on Restasis. After the recruitment patients, an additional 25 consecutive patients showing no symptoms or signs of DED or MGD were then recruited into the study to serve as the control group.

An individual other than the ophthalmologist performing the eye examinations administered the 2 questionnaires. The surveys were not performed in the presence of the examining ophthalmologist, and the answers were unknown to the examining ophthalmologist. The OSDI Dry Eye Questionnaire is well validated and needs no further explanation. 14 The sleep pattern questionnaire is composed of several questions about the patient's sleeping habits and patterns. Specifically, patients were questioned on their most common sleep position (back, right side, left side, alternating sides, stomach with right side down, or stomach with left side down) (Fig. 1).

\section{Distribution of Sleep Position \#1}

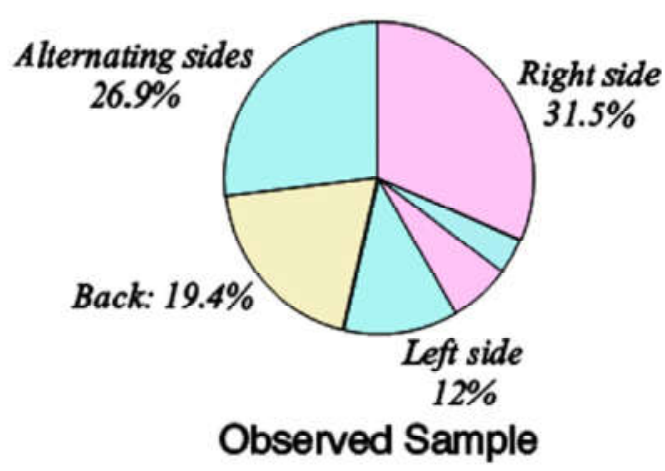

Figure 1 Distribution of patients' primary sleep positions.

Patients were then questioned on how many hours they slept in a typical night, whether they had difficulty sleeping, whether they tended to toss and turn in their sleep, how many times they typically wake up in a given night, and whether they wore contact lenses. In addition, they were asked whether they have ever been diagnosed for DED or MGD or FES (Table 1).

Data were collected and tabulated using Microsoft Word and Microsoft Excel. Statistical analysis was performed on the data set. Analysis of variance (ANOVA) was used to statistically compare the different sleep positions with each variable (FCS, LGS, Schirmer 1 value, etc). T tests were performed to compare between 2 sleep positions and pooled against the supine position. Statistical significance was assumed for $\mathrm{P}, 0.05$.

\section{RESULTS}

One hundred twenty-five subjects were included in the study. An analysis of sleep positions showed that $19.4 \%$ slept on their back, $31.5 \%$ slept on their right side, $8.8 \%$ slept on their stomach, $12 \%$ slept on their left side, and $26.9 \%$ slept on alternating sides (Fig.1). Figure 2 highlights the demographics in our study, which showed similarities to the estimated opulation in the United States.15 In addition, age distribution showed a mean age of 56 years, with the age range of patients from 17 to 89 years (Fig 3).

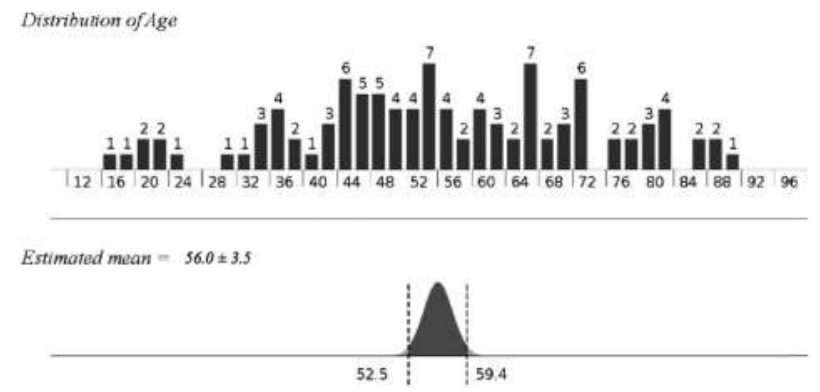

Figure 3 Distribution of age of patients in this study. Estimated mean was found to be 56.0 with an SD of 3.5. 
We organized the data points in categories. The first was the OSDI dry eye questionnaire, which is a 12-question survey graded on a scale of 0 to 100 . The survey is meant to characterize each patient's dry eye symptoms. OSDI symptoms were analyzed based on the patient's sleep pattern. In patients who slept on their back, the average OSDI score was 26.6. In patients who slept on alternating sides, the average OSDI score was 28.0. In patients who slept on their left side, the average OSDI score was 36.4. In patients who slept on their right side, the average OSDI score was 34.2 (Table 1).

\begin{tabular}{|c|c|c|c|c|c|c|c|c|c|}
\hline $\begin{array}{l}\text { Skyp } \\
\text { Pastions }\end{array}$ & Patients & 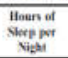 & 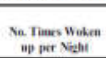 & $\begin{array}{l}\text { Paticat Liag } \\
\text { Cuatad Lreses }\end{array}$ & $\begin{array}{l}\text { OSD } \\
\text { Soury }\end{array}$ & 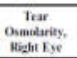 & 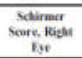 & 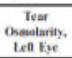 & 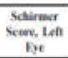 \\
\hline beat & 21 & 6.7 & 2000 & 1 & 26.7 & 3034 & 138 & 249.1 & 123 \\
\hline $\begin{array}{l}\text { Alemating } \\
\text { sulo }\end{array}$ & $\approx$ & 67 & 24 & 1 & 280 & 2989 & 126 & 3014 & 12 \\
\hline Let & 13 & is & 23 & 0 & 364 & 3074 & 121 & 3015 & 112 \\
\hline Right & 34 & 6.8 & 2.1 & 2 & 342 & 3016 & 134 & 306.2 & 13.5 \\
\hline
\end{tabular}

Using $\mathrm{x} 2$, when pooling all the sleep positions and comparing them with the supine position, the P value was, 0.05 (Table 1). In the control group, the average OSDI score was 5.2.

MGD has also been shown to be of importance when looking at the ocular surface; however, in our study, we did not find any statistical significance when comparing patient's MGD staging with the patient's sleep position $(\mathrm{P}=0.98)$. When looking at MGD abnormality and inspissation, there was also no statistical significance when comparing patients who slept on their back with those who slept on their side $(\mathrm{P}=0.14)$ (Table 2). Perhaps a larger study may have reached statistical significance.

LGS is important in assessing and treating DED. LGS stains dead or devitalized cells, allowing the clinician to be specific in the location and severity of DED.17-19 Patients who slept on their back had an LGS score of 4.4. Patients who slept on alternating sides had an LGS score of 4.9. Patients who slept on their right side had an LGS score of 5.6. Last, those patients who slept on their left side had an LGS score of 9.7. Using ANOVA, LGS was found to be statistically significant with $\mathrm{P}=0.005$ when comparing the left side with supine, and comparing pooled sleep positions with supine was $\mathrm{P}, 0.05$. In addition, FCS was also used to assess the patient's dry eye state. The FCS score for patients who slept on their back was 1.4 , alternating sides was 2 , right side 2.7 , and left side 3.7 (Table 2). When comparing each of the sleep positions together with the supine position, FCS was not significant with an ANOVA of $\mathrm{P}=0.0626$ (Table 2).

TABLE 2. Lissamine Staining, Fluorescein Staining, and MGD Staging Scores Based on Patients' Sleep Position

\begin{tabular}{lcccc}
\hline $\begin{array}{l}\text { Sleep } \\
\text { Position }\end{array}$ & $\begin{array}{c}\text { No. } \\
\text { Patients }\end{array}$ & $\begin{array}{c}\text { Average } \\
\text { LGS Score }\end{array}$ & $\begin{array}{c}\text { Average } \\
\text { Fluorescein } \\
\text { Staining Score }\end{array}$ & $\begin{array}{c}\text { Average MGD } \\
\text { Staging Score }\end{array}$ \\
\hline $\begin{array}{l}\text { Back } \\
\text { Alternating }\end{array}$ & 21 & 4.4 & 1.4 & 1.9 \\
$\quad$ sides & 29 & 4.9 & 2 & 2.1 \\
Left & 13 & 9.7 & 3.7 & 2.8 \\
Right & 34 & 5.6 & 2.7 & 2.3 \\
\hline
\end{tabular}

\section{DISCUSSION}

This is the comprehensive study characterizing the sleep position to dry eye can be changed to analyzing the correlation between the sleep position and dry eye. The only similar study in the literature was an observational study highlighting the sleep position and ocular surface in a high- airflow environment focusing on nocturnal lagophthalmos. 9 That study used the Schirmer test (type not described), fluorescein staining, and slit-lamp examination to characterize the ocular surface. The authors found an increase in corneal epitheliopathy in patients sleeping in the dependent position. This occurred in their study in asociation with nocturnal lagophthalmos. The current study evaluated a broader range of patients with dry eye with a control group, including more patients and implemented several dry eye diagnostic modalities to better characterize the patient's DED and provide a better opportunity for determining possible correlations.

Our OSDI survey was helpful because it showed that patients who slept on their back had fewer symptoms than those in the other sleep positions. Also, looking at the average OSDI score based on the sleep position, the 2 highest averages were found in patients who were sleeping on their left and right sides, whereas the lowest score was found in patients who slept on their back. When right and left sides were compared with the supine position, the $\mathrm{P}$ value was, 0.05 .

In our study, MGD was not statistically significant when looking at different sleep positions. With grading on a 1 to 4 scale, subtle differences were difficult to discern. Anecdotally, certain patients who slept on their side showed significant MGD, leading us to believe that a subset of people may have an increase in MGD when sleeping on their sides. Also, we felt that, going into the study, there was a tendency for the DED to be greater on the side patients were sleeping on, but this was not proven, as the data were not statistically significant.

Fluorescein has been universally used to characterize DED and highlight epithelial cellular disruption.18 This stain helped to correlate the findings with LGS in determining the extent of ocular surface disease. In patients who slept on their backs, the FCS was lowest when compared with the other sleep positions, but this failed to reach statistical significance. This could likely be due to many patients having a small amount of nocturnal lagophthalmos exposing the conjunctiva but not the cornea or having a positive Bell phenomenon, which could protect the cornea, reducing the amount of staining that we encountered.

LGS was instrumental in helping to characterize the extent of dry eye in each patient. Patients who slept on either the right or left side as their primary position experienced increased LGS compared with patients who slept on their back. The ANOVA also helped to show a statistically significant correlation $(\mathrm{P}, 0.05)$ between pooled data of the different sleep positions when using LGS as a diagnostic tool and highly significant when comparing left-sided sleeping with supine $(\mathrm{P}=0.005)$. These findings point to a correlation in the ocular surface to differences in sleep habits. We theorize that patients who sleep on their sides or stomach have a higher likelihood of creating chronic mechanical pressure and/or microtrauma to the eyelids, whether it is with the patient's hand, pillow, or bedding. In addition, this microtrauma to the lid may affect nocturnal lagophthalmos, resulting in mild exposure to the conjunctiva more than the cornea, leading to increased LGS staining and less effect on fluorescein staining, especially in patients with a good Bell phenomenon. 
Our findings dovetail with studies that have linked FES to keratoconus. 10 Many patients with FES also have obstructive sleep apnea and chronic ocular surface disease. Thus, at night, these eyelids can evert and lead to direct contact of the eye with bedding, hand, or knuckles.20 Over time, this can lead to a decrease in structural integrity of the eyelid and cornea leading to keratoconus. We theorize a similar process in that patients who do not sleep on their back are prone to this continuous microtrauma, contributing to their dry eye and MGD.

There are several shortcomings in this study. The various sleep positions in the study argues for a larger study group with greater power allowing for more parameters of our study to achieve greater statistical significance. For example, we had 24 patients who slept solely on their right side yet only 13 patients who preferred sleeping on their left side. These numbers lacked the power necessary to achieve better statistical significance. Interestingly, this tendency for sleeping on the right side has been observed in previous studies that have shown preference to the right side as opposed to the left side.7,9

Additional diagnostic testing such as measurements of tear film thickness, meibography, and lipid layer thickness may have proven useful. This would allow a more quantitative method in deciphering MGD. Last, certain patients found difficulty in deciding their favored sleep position. In addition, it is difficult to assess whether a patient changes position throughout the night. Future studies may indeed require using a sleep center to better characterize with certainty each patient's sleep position. 21

In conclusion, we present the findings of this study to support the consideration that the sleep position may be a factor in a patient's dry eye and MGD. We hope that the knowledge of this additional finding may aid physicians in treating patients with DED, MGD, or other ocular surface problems, as changing from a dependent sleeping position to a supine position may lead to improvement of their patient's disease.

\section{References}

1. Gutgesell VJ, Stern GA, Hood Cl. Histopathology of meibomian gland dysfunction. Am $J$ Ophthalmol. 1982;94:383-387.

2. Nelson JD, Shimazaki J, Benitez-del-Castillo JM, et al. The international workshop on meibomian gland dysfunction: report of the definition and classification subcommittee. Invest Ophthalmol Vis Sci. 2011;52:1930-1937.

3. Wu H, Wang $\mathrm{Y}$, Dong $\mathrm{N}$, et al. Meibomian gland dysfunction determines the severity of the dry eye conditions in visual display terminal workers. PLoS One. 2014;9:e105575.

4. Schaumberg DA, Nichols JJ, Papas EB, et al. The international workshop on meibomian gland dysfunction: report of the subcommittee on the epidemiology of, and associated risk factors for, MGD. Invest Ophthalmol Vis Sci. 2011;52:1994-2005.
5. Braich PS, Howard MK, Singh JS. Dyslipidemia and its association with meibomian gland dysfunction. Int Ophthalmol. 2016; 36:469-476.

6. Gordon SJ, Grimmer KA, Trott P. Understanding sleep quality and walking cervicothoracic symptoms. Int $J$ Allied Health Sci Pract. 2007; 5: 1-8.

7. De Koninck J, Lorrain D, Gagnon P. Sleep positions and positions shifts in five age groups: an ontogenetic picture. Sleep. 1992; 15:143-149.

8. Gordon SJ, Grimmer KA, Trott P. Self-reported versus recorded sleep position: an observational study. The Internet Journal of Allied Health Services and Practice. 2004; 2: Article 7.

9. Gauba V, Curtiz ZJ. Sleep position and the ocular surface in a high airflow environment. Saudi $J$ Ophthalmol. 2014; 28:66-68.

10. Pihlblad MS, Schaefer DP. Eyelid laxity, obesity, and obstructive sleep apnea in keratoconus. Cornea. 2013; 32:1232-1236.

11. Donnenfeld ED, Perry HD, Gibralter RP, et al. Keratoconus associated with floppy eyelid syndrome. Ophthalmology. 1991;98:1674-1678.

12. Fowler AM, Dutton JJ. Floppy eyelid syndrome as a subset of lax eyelid conditions: relationships and clinical relevance (an ASOPRS thesis). Ophthal Plast Reconstr Surg. 2010; 26:195-204.

13. Bron AJ, Tiffany JM. The contribution of meibomian gland to dry eye disease. Ocul Surf. 2004; 2:149-165.

14. Cuevas M, González-García MJ, Castellanos E, et al. Correlations among symptoms, signs, and clinical tests in evaporative-type dry eye disease caused by Meibomian gland dysfunction (MGD). Curr Eye Res. 2012; 37:855-863.

15. Schiffman RM, Christianson MD, Jacobsen G, et al. Reliability and validity of the ocular surface disease index. Arch Ophthalmol. 2000; 118: 615-621.

16. Population Distribution by Race/Ethnicity [database online]. Menlo Park, CA: Henry J. Kaiser Family foundation; 2014.

17. Machado LM, Castro RS, Fontes BM. Staining patterns in dry eye syndrome: rose bengal versus lissamine green. Cornea. 2009; 28: 732-734.

18. Cantor L, Rapuano C, Cioffi G, et al. Examination techniques for the external eye and Cornea. In: Basic and Clinical Science Course. External Disease and Cornea. San Francisco, CA: American Academy of Ophthalmology; 2014-2015:16-18.

19. Lemp M, Bron A, Baudouin C, et al. Tear osmolarity in the diagnosis and management of dry eye disease. Am J Ophthalmol. 2011; 151:792-798.e1.

20. Fowler AM, Dutton JJ. Floppy eyelid syndrome as a subset of lax eyelid conditions: relationships and clinical relevance (An ASOPRS thesis). Ophthal Plast Reconstr Surg. 2010; 26:195-204.

21. De Koninck J, Gagnon P, Lallier SR. Sleep positions in the young adult and their relationship with the subjective quality of sleep. Sleep. 1983; 6:52-59. 